TG, total cholesterol CHOL, LDL-CHOL) and blood pressure (BP) in both genders is well known. Some authors (see Sinalko 2004) even documented a constant, progressive increase of $\mathrm{BL}$ and $\mathrm{BP}$, proportional to IRHOMA values.

Method: 288 patients with IR-HOMA $>2 \cdot 5$ (F 160, M 128), out of 683 overweight/obese ones, aged 9-14 years (average age $=11.09$ (SD 2.62) years) were divided into seven IR-HOMA and one unit stepped groups. Every group's BL and BP were confronted with the groups around and with the opposite gender's corresponding group. Statistical analysis: Student's $t$ test and parametric/ non-parametric correlation tests.

Results: In both genders TG decrease $(P<0.05)$ in IRHOMA groups $1-3$, then increase $(P<0 \cdot 05)$; F/M CHOL increases only in groups 6 and $7(P<0 \cdot 05)$. Among $\mathrm{F}$,
LDL-CHOL $(P<0 \cdot 05)$ and $\mathrm{BP}(P<0 \cdot 01)$ also increase in groups 6 and 7 , while among M, LDL-CHOL is increased only in some IR-HOMA groups $(1,3,6)$ and BP increases in group 5 (all $P<0.05$ ). Pairing opposite gender groups, $\mathrm{BL}$ and BP differences are significant in single groups, not at large. Parametric/non-parametric correlations were non-significant.

Conclusions: Notwithstanding our large data allow a quite accurate comparison of metabolic parameters, a progressive, much less constant, increase of any BL or $\mathrm{BP}$, proportional to IR-HOMA increase, could not be demonstrated. Our contribution outlines once more that BL, BP and IR-HOMA are undoubtedly bound, but more factors that IR alone (like genetics, overweight degree and hyperhomocysteinemia) presumably influence this link.

\title{
31 - C-reactive protein: a marker of adiposity or cardiometabolic comorbidities of paediatric obesity?
}

\author{
Andreia Teles ${ }^{1}$, Carla Rego ${ }^{1,2}$, Claudia Dias $^{3}$ and Joao-Tiago Guimaraes ${ }^{4}$
}

${ }^{1}$ Nutrition Unit, Pediatrics Department \& Clinical Pathology Department, Hospital S. Joao, University of Porto, Portugal: ${ }^{2}$ Pediatrics Department, University of Porto, Portugal: ${ }^{3}$ Biostatistics and Medical Informatics (CINTESIS), University of Porto, Portugal: ${ }^{4}$ Biochemistry Department, Faculty of Medicine, University of Porto, Portugal

Background/aims: Childhood obesity is a public health problem. The association between obesity and low-grade inflammation is well established. Our aim is to evaluate the association between C-reactive protein (CRP) and cardiometabolic comorbidities in paediatric obesity.

Material and method: Obese children/adolescents with nutritional obesity followed in our outpatient clinic ( $n$ 354) were included. Duration of disease (years), BMI $Z$ score (Center for Disease Control), percentage of fat mass (dual energy X-ray absorptiometry) and waist circumference were evaluated. Blood pressure, lipid profile and CRP were measured and homeostasis model assessment-insulin resistance (HOMA-IR) was calculated.

Results: The mean chronological age was $10 \cdot 1$ years (SD $3 \cdot 2 ; \min =1 \cdot 7 ; \max =16 \cdot 9)$ with no differences between gender. Same data related to descriptive analyses can be observed in Table 1. CRP was positive and significantly correlated with BMI $Z$-score $(r=0 \cdot 271 ; P<0 \cdot 001)$, \%fat mass $(r=0.366 ; P<0.001)$ and waist circumference $(r=0 \cdot 198 ; P<0 \cdot 001)$. A strong positive correlation was observed between CRP and fat mass, even for short duration of disease $(<2$ years: $r=0.731 ; P<0 \cdot 001)$. No correlations were observed between CRP and lipid profile variables (total, HDL- and LDL-cholesterol, Apo lipoproteins $\mathrm{A} 1$ and $\mathrm{B}$ and triglycerides), systolic and diastolic blood pressure and HOMA-IR, independently of duration of disease.

Conclusions: Magnitude of obesity and adiposity as also intraabdominal fat deposition are predictors of early expression of low-grade inflammation. CRP seems not to be a sensitive/early marker of cardiometabolic comorbidity of paediatric obesity.

\begin{tabular}{|c|c|c|c|c|c|c|c|}
\hline & \multicolumn{2}{|c|}{ Total ( $n$ 354) } & \multicolumn{2}{|c|}{ Females ( $n$ 182) } & \multicolumn{2}{|c|}{ Males ( $n$ 172) } & \multirow[b]{2}{*}{$P$} \\
\hline & Mean & SD & Mean & SD & Mean & SD & \\
\hline BMI Z-score & $4 \cdot 1$ & $1 \cdot 7$ & $4 \cdot 0$ & $1 \cdot 7$ & $4 \cdot 2$ & $1 \cdot 8$ & 0.465 \\
\hline Waist (\%90th Pc) & $117 \cdot 7$ & $12 \cdot 4$ & $118 \cdot 2$ & $15 \cdot 9$ & $116 \cdot 4$ & $11 \cdot 4$ & 0.076 \\
\hline$\%$ Fat mass - (DXA) & $45 \cdot 8$ & $6 \cdot 1$ & $47 \cdot 2$ & $5 \cdot 7$ & $44 \cdot 3$ & $6 \cdot 2$ & 0.002 \\
\hline CRP & 0.31 & $0 \cdot 4$ & 0.32 & 0.4 & $0 \cdot 31$ & $0 \cdot 4$ & 0.581 \\
\hline
\end{tabular}

CRP, C-reactive protein. 